\title{
Prognostic Value of CD74 and HLA-DR Expressions in Invasive Ductal Breast Cancer
}

\author{
Muhittin Yaprak¹, Gülgün Erdogan², Gulbin Aricic ${ }^{3}$, Barıs Ozcan ${ }^{4}$, Ayhan Mesci ${ }^{1}$, \\ Ayhan Dinckan1, Okan Erdogan', Cumhur Arici ${ }^{*}$ \\ ${ }^{1}$ Department of General Surgery, Akdeniz University School of Medicine, Antalya, Turkey \\ ${ }^{2}$ Department of Pathology, Akdeniz University School of Medicine, Antalya, Turkey \\ ${ }^{3}$ Department of Anesthesiology, Akdeniz University School of Medicine, Antalya, Turkey \\ ${ }^{4}$ Department of General Surgery, Medstar Antalya Hospital, Antalya, Turkey \\ Email: muhittin.yaprak@gmail.com, gerdogan@akdeniz.edu.tr, gulbinarici@yahoo.com, \\ barisozcan2004@yahoo.com, drayhanmesci@yahoo.com, adinckan@akdeniz.edu.tr, \\ oerdogan@akdeniz.edu.tr, ${ }^{*}$ cumarici@yahoo.com
}

Received 8 July 2015; accepted 25 July 2015; published 28 July 2015

Copyright (C) 2015 by authors and Scientific Research Publishing Inc.

This work is licensed under the Creative Commons Attribution International License (CC BY). http://creativecommons.org/licenses/by/4.0/

(c) () Open Access

\section{Abstract}

Introduction: Despite the presence of many prognostic and predictive factors, overtreatment remains a major problem in patients with breast cancer. The aim of this study is to investigate the effects of CD74 and HLA-DR expressions on the prognosis of patients who have had a mastectomy for the treatment of breast cancer. Materials and Methods: A retrospective search of medical records was carried out for patients who had surgery for breast cancer at the Department of General Surgery, Akdeniz University School of Medicine, between March 1984 and November 1999. Patients with regular follow-up and necessary data for the study (i.e. patients' demographics, pathology results, and treatment characteristics) were included in the study. Paraffin blocks of tumor specimens were re-examined with immunohistochemical methods in March 2010 to determine the extent of CD74 and HLA-DR expression and the level of tumor infiltrating lymphocyte (TILs). Results: The mean age and the median duration of follow-up for the 41 participants were 48.29 \pm 11.86 years and 125 months (range 115 to 135 months), respectively. Disease-free survival (DFS) in CD74 negative subjects was better than in CD74 positive patients, but the difference was not statistically significant $(p=0.75)$. Similarly, HLA-DR negative and HLA-DR positive groups showed no statistically significant differences in terms of DFS $(p=0.81)$. Conclusion: There were positive but insignificant correlations with increased expression of CD74, decreased expression of HLA-DR, and TILs levels. Further studies involving larger sample sizes may provide more insight into these associations.

*Corresponding author.

How to cite this paper: Yaprak, M., Erdogan, G., Aricic, G., Ozcan, B., Mesci, A., Dınckan, A., Erdogan, O. and Arici, C. (2015) Prognostic Value of CD74 and HLA-DR Expressions in Invasive Ductal Breast Cancer. Advances in Breast Cancer Research, 4, 71-76. http://dx.doi.org/10.4236/abcr.2015.43008 


\section{Keywords}

\section{Breast Cancer, Prognosis, CD74 Antigen, HLA-DR Antigens}

\section{Introduction}

Breast cancer is the most common type of cancer and a leading cause of cancer mortality among women, both in developed and developing countries, including Turkey [1]. A multitude of prognostic and predictive factors have been identified in patients with breast cancer in order to assist in prognostic predictions and to guide management strategies [2] [3]. However, despite significant advances in our understanding of tumor biology and genetics, most of the patients continue to be overtreated [4] [5], necessitating novel prognostic and predictive parameters to be defined to further refine our treatment strategies to reduce unnecessary treatments and the associated morbidity.

Tumor-host interactions are known to play an important role in the growth of neoplasms. In this context, the suggested correlation between tumor infiltrating lymphocyte (TIL) grade and prognosis in many tumor types represents a good example for such interactions [6] [7].

A transmembrane glycoprotein with a variety of immunologic functions, CD74 [8], is associated with human leukocyte antigen-DR (HLA-DR) [major histocompatibility complex (MHC) class II] and represents an important chaperone that regulates antigen presentation for the immune response [9]. It also plays a role in assembly, transport, and loading of peptides onto antigen-presenting cells, and is highly expressed by antigen-presenting cells (APCs), including B cells, monocytes, macrophages and dendritic cells in normal tissues [10]. CD74 expression has also been reported in some malignant tumors [6] [8]-[10]. HLA-DR molecules and CD74 interact in the endoplasmic reticulum. Because of this interaction, binding of HLA-DR molecules to endogenous peptides is prevented and the host immune response to tumor cells is suppressed [8]. Therefore, tumor tissues expressing high levels of CD74 may exhibit a worse prognosis as they are able to escape from the host's defenses [6].

Recent research has focused on the assessment of the prognostic and predictive value of CD74 and HLA-DR expression in various types of cancers including colorectal, gastric, pancreatic and breast cancer [6] [8] [9] [11]. For instance, Jiang et al. have found an inverse association between the frequency of TILs and CD74 expression, suggesting less immunogenicity and less stimulation of host immune system with increased expression of CD74 [6]. Naga et al. established the independent prognostic value of CD74 expression in pancreatic ductal adenocarcinoma [8]. In another study assessing the association between CD74 expression and gastric cancer prognosis along with the clinical and pathological characteristics of CD74-positive gastric cancers, Ishigami et al. proposed a useful prognostic role for CD74 expression as an HLA class II-associated prognostic indicator in gastric cancer [9].

In this study, the effects of CD74 and HLA-DR expressions on the prognosis of patients undergoing mastectomy for invasive ductal breast cancer was investigated.

\section{Materials and Methods}

A retrospective search of medical records was carried out for patients who had surgery for breast cancer at the Department of General Surgery, Akdeniz University School of Medicine (a tertiary care facility in Antalya, Turkey), between March 1984 and November 1999. A total of 41 patients who have completed the 10-year follow-up period with regular attendance to postoperative follow-up visits and adequate data in medical records on the following parameters were included in the study: demographic; pathologic and treatment characteristics including age; duration of follow-up; menopausal status; tumor localization; operation technique; tumor size; lymph node metastasis; nuclear grade; histological grade; hormone receptor status; chemotherapy; radiotherapy; hormonal therapy; presence of local-regional recurrence (LRR); and systemic recurrence (SR). Patients were categorized into two groups as follows: poor prognosis group with LRR and/or SR; and favorable prognosis group with no recurrence.

Patients' paraffin-embedded blocks of tumor tissue were retrieved from the Department of Pathology, Akdeniz University Hospital, and were re-examined using immunohistochemical methods in March 2010. Tissue cross-sections of 4 - $5 \mathrm{mM}$ (micrometer) thickness prepared from paraffin blocks were immunohistochemically 
treated with Anti-CD74 (clone 1N2, 1/100, Lab vision, Neomarker) and anti-HLA-DR (Clone CR3/43, Dako, 1/50) antibodies using the streptoavidin-biotin complex method. CD74 and HLA-DR expression as well as lymphocyte infiltration were assessed in cancer tissues (Figure 1(A) and Figure 1(B)).

\section{Evaluation of Immunohistochemical Staining}

A semi-quantitative assessment was performed using light microscopy. Presence of cytoplasmic staining was considered positive for CD74 and HLA-DR. The level of HLA-DR and CD74 expression and grade of lymphocyte infiltration were evaluated according to Jiang's classification [6]. Accordingly, presence of $\geq 10 \%$ positive tumor cell staining was considered positive for CD74 or HLA-DR and presence of $<10 \%$ positive tumor cell staining was considered negative for CD74 or HLA-DR. Similarly, patients with numerous large lymphoid aggregates with frequent germinal centers were defined as TILs positive, while those with no or occasional lymphoid aggregates with rare or absent germinal centers comprised the TILs negative group [6] [9]. Then, the association between the results of these assessments and prognostic data obtained from medical records was examined.

SPSS (Statistical Package for Social Sciences) for Windows version 13.0 (SPSS inc. Chicago, IL) program was used for statistical analyses. Chi-square test and Mann-Whitney test were used to analyze the correlation between CD74 and HLA-DR expression and clinical/pathological parameters. The cumulative survival was calculated by Kaplan-Meier method and analyzed by the Log rank test. A $P$ level of less than 0.05 was considered statistically significant.

The study protocol was approved by the Local Ethics Committee, Akdeniz University.

\section{Results}

The mean age and median follow-up for 41 patients were $48.29 \pm 11.86$ years and 125 months (range 115 to 135 months), respectively. Only $24 \%$ of the patients had Stage I breast cancer. Adjuvant systemic and radiation therapy was administered to $83 \%$ and $63 \%$ of the subjects, respectively. Hormonal therapy was given to 33 (80\%) hormone receptor positive patients. Systemic recurrence (SR) and loco-regional recurrence (LRR) were detected in 18 (43.9\%) and 3 (7\%) patients during the follow-up period.

Immunohistochemical staining showed a CD74, HLA-DR and TILs positivity rate of 53.7\%, 43\%, and 41.5\%, respectively.

The differences in HLA-DR expression ( $\mathrm{p}=0.67)$ and TILs infiltration $(\mathrm{p}=0.06)$ between the CD74 positive and negative groups were not statistically significant. Similarly, there were no significant differences between the HLA-DR positive and negative subjects in terms of lymphocyte infiltration ( $p=0.73$ ).

Although the disease-free survival (DFS) was better in CD74 negative subjects than in CD74 positive subjects, the difference was not statistically significant $(\mathrm{p}=0.75$, Figure 2(A)). Similarly, HLA-DR negative and HLADR positive patients did not differ significantly in terms of DFS $(p=0.81$, Figure 2(B)). Also, although DFS
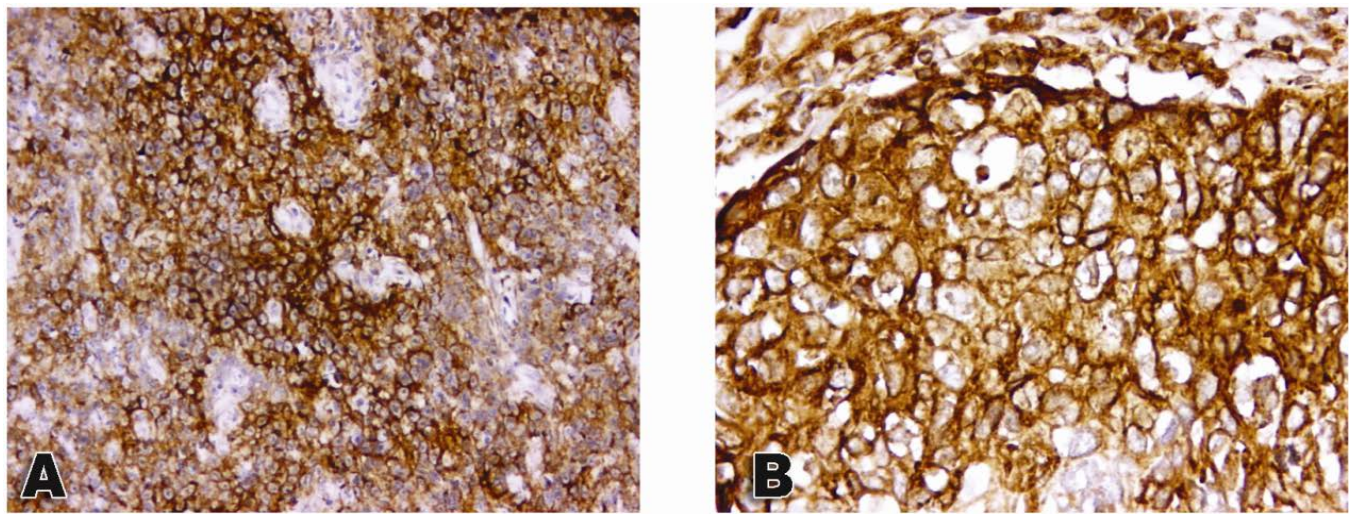

Figure 1. Microscopic sections showing immunohistochemical staining of HLA-DR in invasive ductal carcinoma, DAB $\times 200(\mathrm{~A})$; and immunohistochemical staining of CD74 in invasive ductal carcinoma, $\mathrm{DAB} \times 400(\mathrm{~B})$. Both HLA-DR (A) and CD74 (B) immunoreactivity was identified on the surface and cytoplasm of the tumor cells. Some populations of tumor infiltrating lymphocytes were also immunopositive for those markers. 


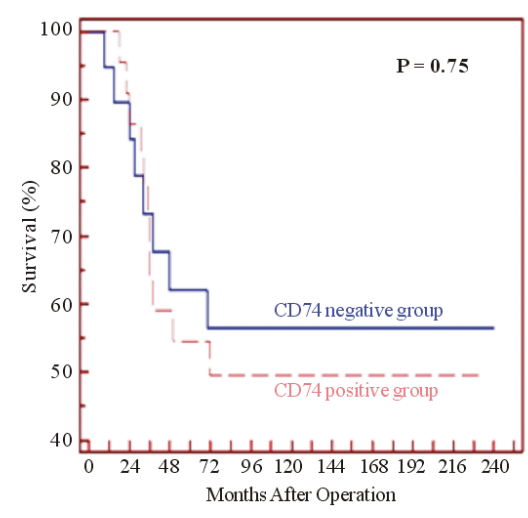

(A)

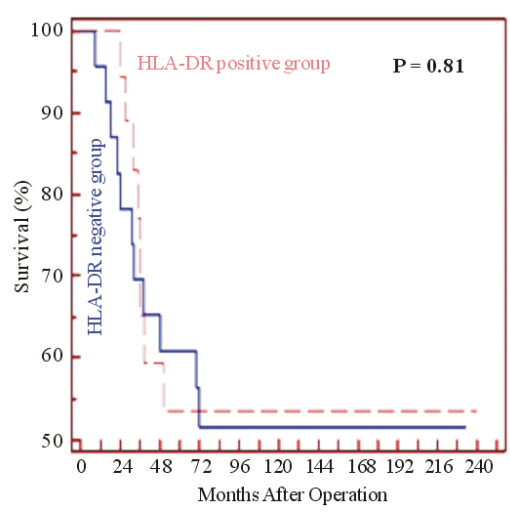

(B)

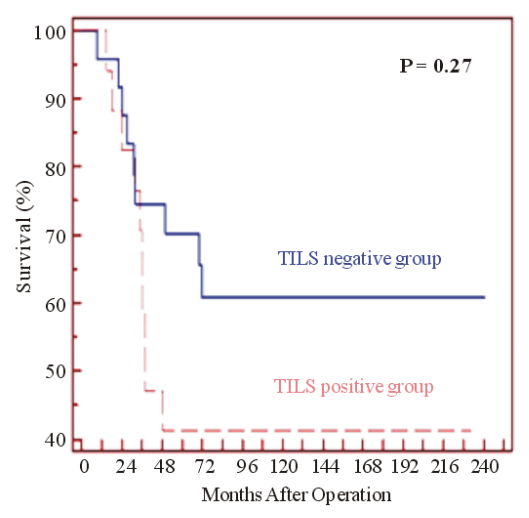

(C)

Figure 2. Comparison of CD74 expression rates and disease-free survival (A); HLA-DR expression rates and disease-free survival (B); and TILs infiltration and disease-free survival (C).

was better in TILs negative patients, the difference did not reach statistical significance when compared with TILs positive patients $(p=0.27$, Figure $2(C)$ ).

Lymph node metastasis was more frequent in HLA-DR negative patients (69.6\%) than in positives (55.6\%), the difference being insignificant $(\mathrm{p}=0.4)$. However, with respect to the TNM classification, a statistically significant difference was found between the two groups whereby the majority of N3 involvement was in the HLA-DR negative group, while the majority of $\mathrm{N} 2$ involvement was in the HLA-DR positive group $(\mathrm{p}=0.044)$.

Differences between the CD74 negative and positive patients with respect to tumor stage ( $p=0.9)$, axillary lymph node metastasis $(\mathrm{p}=0.2)$, nuclear grade $(\mathrm{p}=0.3)$, histological grade $(\mathrm{p}=0.4)$ and presence of distant metastasis $(\mathrm{p}=0.8)$ were insignificant. Also there were no significant differences between HLA-DR negative and positive groups in terms of tumor stage $(p=0.5)$, nuclear grade $(p=0.2)$, histological grade $(p=0.5)$ and presence of distant metastasis $(\mathrm{p}=0.6)$ (Table 1$)$.

\section{Discussion}

As in many other malignant conditions, prognostic and predictive factors play an important role in determining the optimal management strategy for breast cancer. A prognostic factor may be defined as a measurable variable that correlates with the natural history of disease progression, in the absence of systemic therapies. In contrast, a predictive factor is any measurement associated with response to a specific therapy. Some factors, such as hormone receptors and human epidermal growth factor receptor 2 (HER2/neu) overexpression, are both prognostic and predictive [2] [3]. The major prognostic factor for patients with breast cancer is the presence or absence of axillary lymph node involvement [2]. Tumor size, histological grade, and hormone receptor status are other important prognostic factors related to breast cancer [3]. Estrogen and progesterone receptor content in a breast tumor is a well-established predictive factor for response to endocrine treatment [2] [3]. Patient age, lymphatic/vascular invasion, histological subtype, response to neoadjuvant therapy, and HER2/neu overexpression are other recognized prognostic and predictive factors for breast cancer [2] [3]. In addition to those already established indicators, certain other potential prognostic/predictive factors are also being researched including gene expression profile, urokinase plasminogen activator, bone marrow micrometastasis, p53 gene, cathepsin D, microvessel density, and DNA ploidy analysis [3]. Despite the prognostic and predictive value of the currently utilized parameters, their usefulness in determining the best candidates for adjuvant systemic therapy for breast cancer is still limited.

CD74 is a non-polymorphic glycoprotein involved in various immunological functions such as antigen presentation, inflammation, and the behavior of cancer tissues. Among these, the best known is the regulation of the trafficking of HLA-DR proteins in antigen presenting cells. CD74 expression in tumor cells has been suggested to prevent presentation of tumor antigens by HLA-DR molecules [9]. Consequently, poor antigen presentation to intratumoral T-cells may undermine the host immune defense against tumor cells.

CD74 and HLA-DR expressions have been previously found to influence the prognosis of various types of cancers [6] [8] [9] [11]. In the study by Ishigami et al., the association between CD74 expression and prognosis 
Table 1. Comparison of the CD74 negative and positive, and HLA-DR negative and positive groups in terms of tumor stage, axillary lymph node metastasis, nuclear grade, histological grade, and presence of distant metastasis.

\begin{tabular}{|c|c|c|c|c|c|c|}
\hline & CD74 negative & CD74 positive & & HLA-DR negative & HLA-DR positive & \\
\hline & Group & Group & $\mathbf{P}$ & Group & Group & \\
\hline Tumor stage & & & 0.9 & & & 0.5 \\
\hline $\mathrm{T} 1$ & $4(21 \%)$ & $6(27 \%)$ & & $5(22 \%)$ & $5(28 \%)$ & \\
\hline $\mathrm{T} 2$ & $12(63 \%)$ & 13 (59\%) & & $13(56 \%)$ & $12(67 \%)$ & \\
\hline T3 & $2(11 \%)$ & $2(9 \%)$ & & $3(13 \%)$ & $1(5 \%)$ & \\
\hline $\mathrm{T} 4$ & $1(5 \%)$ & $1(5 \%)$ & & $2(9 \%)$ & 0 & \\
\hline \multicolumn{7}{|l|}{ Axillary lymph } \\
\hline Node metastasis & & & 0.2 & & & 0.4 \\
\hline Positive & 10 (53\%) & $16(73 \%)$ & & $16(70 \%)$ & $10(56 \%)$ & \\
\hline Negative & $9(47 \%)$ & $6(27 \%)$ & & $7(30 \%)$ & $8(44 \%)$ & \\
\hline Nuclear grade & & & 0.3 & & & 0.2 \\
\hline Grade 1 & $5(26 \%)$ & $6(27 \%)$ & & $8(35 \%)$ & $3(17 \%)$ & \\
\hline Grade 2 & $11(58 \%)$ & $16(73 \%)$ & & $12(52 \%)$ & 15 (83\%) & \\
\hline Grade 3 & $3(16 \%)$ & 0 & & $3(13 \%)$ & 0 & \\
\hline Histological grade & & & 0.4 & & & 0.5 \\
\hline Grade 1 & $2(11 \%)$ & 0 & & 0 & $1(6 \%)$ & \\
\hline Grade 2 & $12(63 \%)$ & $17(77 \%)$ & & $16(70 \%)$ & $13(72 \%)$ & \\
\hline Grade 3 & $5(26 \%)$ & $5(23 \%)$ & & $7(30 \%)$ & $4(22 \%)$ & \\
\hline Distant metastasis & & & 0.8 & & & 0.6 \\
\hline Positive & $8(42 \%)$ & $10(45 \%)$ & & $10(43 \%)$ & $8(44 \%)$ & \\
\hline Negative & $11(58 \%)$ & $12(55 \%)$ & & $13(57 \%)$ & $10(56 \%)$ & \\
\hline
\end{tabular}

[9] was examined in 126 patients with gastric cancer. Of these patients, 48 were CD74 positive and there was a negative correlation between CD74 expression and the depth of tumor invasion as well as the patient's clinical stage. In addition, CD74 expression negatively correlated with HLA-DR expression. Patients with no CD74 expression had significantly better surgical outcomes than CD74-positive subjects $(p<0.05)$. In the present study, CD74 expression also negatively correlated with HLA-DR expression, but the difference was not significant (p $=0.67)$.

In the study by Nagata et al., CD74 expression emerged as an independent prognostic indicator for pancreatic ductal adenocarcinoma [8]. In their study, 47 (69.1\%) and 21 (30.9\%) patients showed level I and level II CD74 expression, respectively. Patients with level II CD74 expression showed a higher rate of lymphatic permeation $(p=0.04)$ and perineural invasion $(p=0.01)$ compared with those having level I expression. In addition, patients with level I CD74 expression had a significantly better survival rate than those with level II $(p=0.003)$. Similarly, in our study, survival rate was better in CD74 negative subjects, although the difference was not statistically significant $(\mathrm{p}=0.75)$. In Jiang et al.'s study, CD74 expression appeared to increase the risk of progression from low- to high-grade colon neoplasms and this effect was most marked in the poorly differentiated carcinomas [6]. These investigators also showed a negative association between CD74 expression and frequency of TILs. A similar observation was made in our study, although this association was not statistically significant $(p=0.06)$.

\section{Conclusion}

In our study, majority of TNM N3 or N2 lymph node involvement occurred in HLA-DR negative and positive 
patients, respectively and this difference was significant $(p=0.044)$. This observation is supportive of the view that low levels of HLA-DR expression may be associated with insufficient host immune response in tumors with low HLA-DR expression. DFS in CD74 negative and HLA-DR positive patients was better when compared with CD74 positive and HLA-DR negative subjects. Lymph node metastasis was more common in CD74 positive and HLA-DR negative groups in comparison with CD74 negative HLA-DR positive subjects. Also, TILs negative patients experienced more frequent metastases than TILs positive patients. This piece of information can generally be regarded as supportive of our view on the effect of these factors on prognosis. However, statistical significance could not be reached, probably due to the inadequate sample size. Further studies with larger sample sizes providing higher statistical power may help elucidate the role of these factors in preventing overtreatment in patients with breast cancer.

\section{Conflict of Interest}

There is no conflict of interest.

\section{References}

[1] Ozmen, V. (2008) Breast Cancer in the World and Turkey. Journal of Breast Health, 4, 6-12.

[2] Aebi, S., Davidson, T., Gruber, G. and Cardoso, F. (2011) Primary Breast Cancer: ESMO Clinical Practice Guidelines for Diagnosis, Treatment and Follow-Up. Annals of Oncology, 22, vi12-vi24. http://dx.doi.org/10.1093/annonc/mdr371

[3] Subramaniam, D.S. and Isaacs, C. (2005) Utilizing Prognostic and Predictive Factors in Breast Cancer. Current Treatment Options in Oncology, 6, 147-159. http://dx.doi.org/10.1007/s11864-005-0022-1

[4] Weigel, M.T. and Dowsett, M. (2010) Current and Emerging Biomarkers in Breast Cancer: Prognosis and Prediction. Endocrine-Related Cancer, 17, R245-R262. http://dx.doi.org/10.1677/erc-10-0136

[5] Knauer, M., Cardoso, F., Wesseling, J., Bedard, P.L., Linn, S.C., Rutgers, E.J.T. and van’t Veer, L.J. (2010) Identification of a Low-Risk Subgroup of HER-2-Positive Breast Cancer by the 70-Gene Prognosis Signature. British Journal of Cancer, 103, 1788-1793. http://dx.doi.org/10.1038/sj.bjc.6605916

[6] Jiang, Z., Xu, M., Savas, L., LeClair, P. and Banner, B.F. (1999) Invariant Chain Expression in Colon Neoplasms. Virchows Archiv, 435, 32-36. http://dx.doi.org/10.1007/s004280050391

[7] Ohtani, H. (2007) Focus on TILs: Prognostic Significance of Tumor Infiltrating Lymphocytes in Human Colorectal Cancer. Cancer Immunity, 7, 4.

[8] Nagata, S., Jin, Y.F., Yoshizato, K., Tomoeda, M., Song, M., Iizuka, N., et al. (2009) CD74 Is a Novel Prognostic Factor for Patients with Pancreatic Cancer Receiving Multimodal Theraphy. Annals of Surgical Oncology, 16, 25312538. http://dx.doi.org/10.1245/s10434-009-0532-3

[9] Ishigami, S., Natsugoe, S., Tokuda, K., Nakajo, A., Iwashige, H., Aridome, K., et al. (2001) Invariant Chain Expression in Gastric Cancer. Cancer Letters, 168, 87-91. http://dx.doi.org/10.1016/S0304-3835(01)00503-1

[10] Zheng, Y.X., Yang, M., Rong, T.T., Yuan, X.L., Ma, Y.H., Wang, Z.H., Shen, L.S. and Cui, L. (2012) CD74 and Macrophage Migration Inhibitory Factor as Therapeutic Targets in Gastric Cancer. World Journal of Gastroenterology, 18, 2253-2261. http://dx.doi.org/10.3748/wjg.v18.i18.2253

[11] Lin, X., Wang, X., Capek, H.L., Simone, L.C., Tuli, A., Morris, C.R., et al. (2009) Effect of Invariant Chain on Major Histocompatibility Complex Class I Molecule Expression and Stability on Human Breast Tumor Cell Lines. Cancer Immunology, Immunotherapy, 58, 729-736. http://dx.doi.org/10.1007/s00262-008-0595-1 